\title{
Synthesis and controlled release properties of 2,4-dichlorophenoxy acetate-zinc layered hydroxide nanohybrid
}

\begin{abstract}
Direct reaction of $\mathrm{ZnO}$ with 2,4-dichlorophenoxyacetic acid (24D) solutions of different concentrations allows obtaining new organic-inorganic nanohybrid materials formed by intercalation of 24D into interlayers of zinc layered hydroxide (ZLH). XRD patterns show a progressive evolution of the structure as 24D concentration increases. The nanohybrid obtained at higher 24D concentration (24D-ZLH(0.4)) reveals a well ordered layered structure with two different basal spacings at $25.2 \AA$ and $24 \AA$. The FTIR spectrum showing the vibrations bands of the functional groups of 24D and of the ZLH confirms the intercalation. SEM images are in agreement with the structural evolution observed by XRD and reveal the ribbon morphology of the nanohybrids. The release studies of 24D showed a rapid release of $94 \%$ for the first 100 min governed by the pseudo-second order kinetic model.
\end{abstract}

Keyword: 2,4-dichlorophenoxyacetic acid; Zinc layered hydroxide; Nanohybrid; Controlled release. 